\title{
An Adaptive and Fast CFAR Algorithm Based on Multithreading for Ship Detection in SAR Image
}

\author{
Ruifu Wang ${ }^{1,2}, \mathrm{Jie} \mathrm{Li}^{1}$, YingJie $\mathrm{Hao}^{1}$ and Jiagui $\mathrm{Li}^{1}$ \\ ${ }^{1}$ Shandong University of Science and Technology, College Of Geomatics, \\ Qingdao, 266590, China \\ ${ }^{2}$ The Key Lab of Surveying \& Mapping Technology on Island and Reef, SBSM, \\ Qingdao, 266590, China \\ wangruifu@263.net,sdhzjclj01@163.com
}

\begin{abstract}
In order to improve the speed of the ship detection, an adaptive and fast constant false alarm rate (CFAR) algorithm based on multithreading is proposed in this paper for ship detection in synthetic aperture radar(SAR) images. At first, the global threshold is calculated to prescreen the target pixels out rapidly by histogram statistics and CFAR algorithm, and obtain the index matrix. Then, the possible target pixels are detected in the sliding window based on the $K$ distribution by the index matrix, and the multithreading technology is adopted to improve the local detection speed. Finally, the possible ships are screened out by making the four-connected neighborhood area statistics for the target pixels obtained in local detection according to the pixel area of the smallest ship. It is concluded that the accuracy and speed of the algorithm is improved greatly by analyzing the SAR image detection results, and the algorithm is more suitable for application to the ship detection system.
\end{abstract}

Keywords: constant false alarm rate (CFAR), ship detection, synthetic aperture radar (SAR), multithreading technology

\section{Introduction}

With the development of the global Marine technology, the requirements of ship detection are becoming higher and higher on the aspect of real-time and accuracy. So far, a lot of algorithms about target detection have been proposed in the literature. Among these algorithms, the constant false alarm rate (CFAR) detection algorithm is considered to be an important algorithm in SAR image target detection for simple calculation, adaptive threshold and fast detection speed in complex background [1], which has been widely applied to many ship detection systems.

Generally, the CFAR target detection needs choosing appropriate clutter distribution model and detector. The commonly used statistical model includes Gaussian distribution, Rayleigh distribution, Lognormal distribution, Weibull distribution [2], $\mathrm{G}^{0}$ distribution [3], $\mathrm{K}$ distribution [4] and so on in the clutter environment. The commonly used CFAR detector includes [5] cell averaging CFAR (CA-CFAR), order statistic CFAR (OS-CFAR), greatest of CFAR (GO-CFAR), smallest of CFAR (SO-CFAR), and their combination, etc. CA-CFAR detection performs very well in a homogeneous clutter, but poor in the non-homogeneous environment. GO, SO, OS algorithms are lack of universality in non-homogeneous clutter environment [6].In order to improve the universality of the algorithm, Smith and Varshney [7] proposed the VI-CFAR (Variability Index CFAR) from the perspective of adaptively choosing algorithm, which can select the most suitable algorithm dynamically from $\mathrm{CA}, \mathrm{SO}, \mathrm{GO}$ algorithm according to the VI hypothesis test. 
Salazar [8] proposed a notable CFAR algorithm based on Beta-prime distribution (mentioned simply as Salazar algorithm in this paper); Bisceglie [9] put forward a CFARalgorithm to reduce a part of the pixels (mentioned simply as Bisceglie algorithm in this paper), which is well-suited for local area target detection; Lincoln Laboratory proposed the two-parameter CFAR algorithm [10], which is actually based on CA-CFAR with normal distribution model, which improved the computational efficiency significantly; Gui Gao [11] proposed an adaptive and fast CFAR detection algorithm based on AC, which is an improvement of the Salazar algorithm; Wentao An [12] proposed an improved iterative censoring scheme (ICS) for CFAR detectors with two modifications to eliminate the influence of target returns on the estimation of local sea clutter distributions and so on. Through these literatures, we can draw the conclusion that the target detection algorithm based on CFAR is the most extensive and practical. However, so far, there hasn't been any algorithm to meet the real-time requirements of ship detection for military combat.

In order to further explore how to raise the ship detection speed, an adaptive and fast CFAR algorithm for ship detection based on multithreading is proposed in this paper, which is an improvement of the two-parameter CFAR algorithm, which has higher detection efficiency. The rest of paper is arranged as follows. In Section II, the flow of the algorithm is detailed. In Section III, the principle of the fast algorithm is given. In Section IV, the SAR image detection results of the presented algorithm are analyzed. In Section V, this paper is concluded and the prospect of the ship detection is presented.

\section{The Idea and Detailed Flow of Fast Algorithm}

\subsection{The Idea of Fast Algorithm}

The adaptive and fast ship detection algorithm proposed in this paper adopts some ideas in classical CFAR algorithms. (1) The index values in the VI-CFAR [7] can be used in selecting the target pixels in clutter environment, but the index matrix should be obtained by the adaptive threshold rather than the empirical value. (2) The detection effect of Salazar algorithm [8] is better in homogeneous clutter environment and multi-target situations, so local detection should be made for single target pixel in the multi-target situations, to ensure the accuracy of target detection. (3) Bisceglie algorithm [9] removes the possible target pixels in background statistics in order to avoid clutter interference effects, which should be used in local detection. (4) By combining the index values with sliding window, we can screen out whether the target pixels are the clutter pixels or ship pixels.

This paper designs the adaptive and fast detection algorithm based on the above ideas. Firstly, a global detection for the whole SAR image should be made. Make a simple histogram statistics for all pixel values in the SAR image. Then set the first level CFAR value to calculate the global threshold. Finally all possible target pixels are screened out quickly by the index value and marked, which are referred to as the marked pixels in this paper. Secondly, a local detection should be made. Set a sliding window for all marked pixels obtained in global detection, and remove the possible target pixels in clutter window in order to avoid clutter interfering. Then make an accumulated statistics for the rest pixels with the relatively accurate $\mathrm{K}$ distribution model, and set the second level CFAR value to calculate the local threshold. Finally the local detection for target pixels is completed by the comparison of the local threshold and the target pixel values. In the process, we can improve the speed of local detection by multithreading technology. Thirdly, the four-connected neighborhood areas statistics should be made for the target pixels obtained in local detection, and the possible ships are screened out according to the smallest vessel pixel areas. 


\subsection{The Detailed Flow of Fast Algorithm}



Figure 1. The Detection Flow of Fast Algorithm

As is shown in Figure 1, the fast detection process of the algorithm proposed in this paper is summarized, and detailed steps are described as follows.

(1)The global threshold is computed by setting a false alarm rate.

There are two ways to obtain the global threshold (referred to as $T_{\text {low }}$ in this paper). The one way, make a histogram statistics for all pixel values in the whole SAR image to calculate the global threshold by setting a CFAR value. The other way, set a global threshold directly. It stands to reason that the detection effect of the first way is better and more accurate, so we adopt the first way to calculate the global threshold in this paper.

(2) The possible target pixels are screened out to obtain the index matrix.

After the global threshold $\mathrm{T}_{\text {low }}$ is calculated out, every pixel value in SAR image should be compared with the global threshold. The pixel value less than $T_{\text {low }}$ is set to 0 , and the pixel value greater than $T_{\text {low }}$ is set to 1 , and when all pixels are finished, the index matrix is obtained. The pixel whose value is 1 in the index matrix may be the target pixel in the ship, and should be marked, so we refer to them as the marked pixels in this paper.

(3) The SAR image is divided into four regions, and detected by multithreading technology at the same time.

Because the parameters calculation of $\mathrm{K}$ distribution model is complex particularly, the detection speed is very slow when the number of detection targets is too large. The SAR image is divided into four regions in local detection in this paper, and four regions are detected at the same time by multithreading technology.

(4) The sliding window is established based on a marked pixel. 


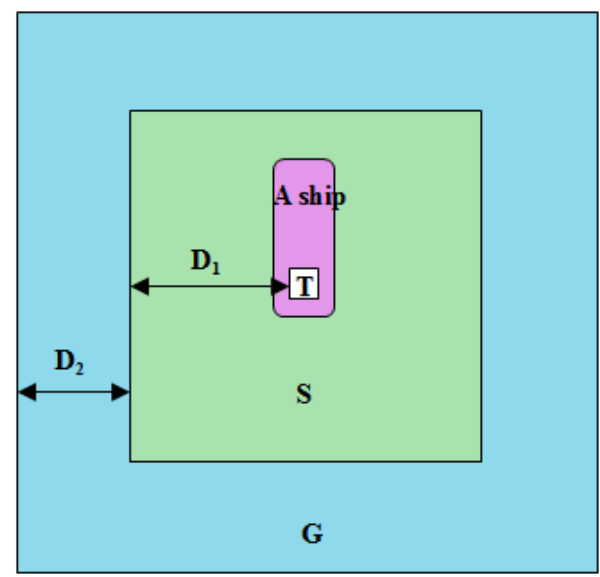

Figure 2. The Sliding Window

As shown in Figure 2, three windows are established based on the marked pixels in the index matrix: the target window ( $\mathrm{T}$ for short), the protected window ( $\mathrm{S}$ for short), the background window ( $\mathrm{G}$ for short).

The target pixel can be set to the target window, and the distance between the edges of the protected window and the edge of the target window can be set to the ship pixel length of 1.5 times, referred to as $D_{1}$. If the maximum ship's length is set to $300 \mathrm{~m}$ in this paper, the solution referred to as $\mathrm{R}$, the ship pixel length referred to as $\mathrm{L}_{\mathrm{P}}$, we can get the formula $L_{P}=\frac{300}{R}, \mathrm{D}_{1}=1.5 \times L_{P}$. After many experiments, we know it is more accurate to set 20000 pixels in the region area between the background window and the protected window in less than $15 \mathrm{~m}$ high resolution, and 1000 pixels in low resolution when making a local detection in the sliding window. If the distance between the edges of the background window and the edge of the target window is referred to as $\mathrm{D}_{2}$, the relationship between $\mathrm{D}_{1}$ and $D_{2}$ is $D_{2}=\frac{\sqrt{\left(2 D_{1}+1\right)^{2}+20000}-\left(2 D_{1}+1\right)}{2}$ in high resolution or $D_{2}=\frac{\sqrt{\left(2 D_{1}+1\right)^{2}+1000}-\left(2 D_{1}+1\right)}{2}$ in low resolution.

(5) The local threshold is computed.

Generally speaking, the distance between two ships is at least twice as long as the ship, in other words, there should be no ships in the background window. So the pixels whose value greater than the global threshold should be removed in background window, to prevent the clutter whose pixel value is higher is taken as ship. Then make a statistic for the rest pixels with $\mathrm{K}$ distribution model, and calculate the local threshold (referred to as $\mathrm{T}_{\mathrm{G}}$ in this paper) in background window. Finally, we can concluded that if $\mathrm{T}>\mathrm{T}_{\mathrm{G}}$, the target pixel is the ship pixel, and if not, the target pixel is clutter pixel by comparing the target pixel value (referred to as $\mathrm{T}$ in this paper) with the local threshold $\mathrm{T}_{\mathrm{G}}$.

(6) The ships are screened out by making the four-connected neighborhood area statistics.

The four-connected neighborhood area statistics are made for the target pixels obtained in local detection, and we can screen out the possible ships according to the smallest vessel pixel area. Generally, the minimum ship is 15 meters long and 4 meters wide, so the minimum ship pixel area formula is $\mathrm{S}_{\min }=\frac{15}{R} * \frac{4}{R}$ in the algorithm. For the formula, the resolution is referred to as $R$ and the minimum ship pixel area is referred to as $S_{\min }$. If its area is less than $S_{\min }$, the four-connected neighborhood region should be removed and the rest regions are the ships detected by the algorithm in this paper. 


\section{The Detection Principle of Fast CFAR Algorithm}

\subsection{K Distribution Model}

By comparing commonly used clutter statistical models, $\mathrm{K}$ distribution model is a good way to describe the heavy-tailed characteristics of the clutter distribution, which can be matched with amplitude distribution of sea clutter well in a wide range, which can meet the requirement of local adaptive detection in many cases. As a result, $\mathrm{K}$ distribution model is adopted to detect ships in this paper.

The probability density function of $\mathrm{K}$ distribution is as follows:

$$
f_{A}(x)=\frac{4}{\Gamma(\mathrm{L}) \Gamma(\gamma)}\left(\frac{L \gamma}{\mu}\right)^{\frac{L+\gamma}{2}} x^{L+\gamma-1} K_{\gamma-L}\left(2 x \sqrt{\frac{L \gamma}{\mu}}\right), x \geq 0
$$

Where $\quad \Gamma(*)=$ Gamma function, $\Gamma(x)=\int_{0}^{+\infty} t^{x-1} e^{-t} d t$

$K_{\gamma-L}=$ the second modified Bessel function of $\gamma-\mathrm{L}$

$\mathrm{L} \square \mathrm{Y} \square \mu=$ three parameters which can be solved by formula

$L+\gamma-1=$ the shape parameter

The shape parameter determines the sharp degree of curve. The smaller whose value is, the more obvious the tail length of $\mathrm{K}$ distribution is. As a result, the $\mathrm{K}$ distribution curve is more suitable to describe the sea clutter.

The expression of three parameters $L, \Upsilon, \mu$ is as follows:

$$
\begin{aligned}
2 \mathrm{k}_{1} & =\log \frac{\mu}{L \gamma}+\Phi_{0}(\mathrm{~L})+\Phi_{0}(\Upsilon) \\
4 \mathrm{k}_{2} & =\Phi_{0}(1, \mathrm{~L})+\Phi_{0}(1, \Upsilon) \\
8 \mathrm{k}_{3} & =\Phi_{0}(2, \mathrm{~L})+\Phi_{0} 2,(\Upsilon)
\end{aligned}
$$

Where $\Phi_{0}(\mathrm{~L})=$ the logarithmic derivative of the gamma function when $x$ is $\mathrm{L}, \psi(x)=\frac{d}{d x} \ln \Gamma(x)=\frac{\Gamma \prime(x)}{\Gamma(x)}$

$\mathrm{K}_{1}=$ the mean value, known parameter

$\mathrm{K}_{2}=$ the square error, known parameter

$\mathrm{K}_{3}=$ three times the variance, known parameter

\subsection{The Basic Principle of CFAR Detection}

1) The principle of the first level CFAR detection

The first level false alarm rate (referred to as $\mathrm{P}_{\mathrm{fa}}$ in the paper) can be set for users, and the histogram probability density function (referred to as $f(x)$ in the paper) is obtained by making a histogram statistic for all pixels in the whole SAR image. The global threshold $\mathrm{T}_{\text {low }}$ is obtained by solving the equation 3.5. If its value in the SAR image is greater than $\mathrm{T}_{\text {low }}$, the pixel is considered as the possible ship pixel, which is different from the surrounding clutter, and if not, the pixel is considered as clutter pixel. The decision rule is as shown in equation 3.6.

$$
P_{\mathrm{fa}}=\int_{T}^{\infty} f(x) d x
$$

The decision rule is as follows. 


$$
\mathrm{T} \begin{cases}>T_{\text {low, }}, & \text { target pixel } \\ <T_{\text {low }}, & \text { clutter pixel }\end{cases}
$$

2) The principle of the second level CFAR detection

The second level CFAR detection of SAR image is completed by combining the sliding windows with the index values. The sliding windows are established based on the index values obtained by the first level CFAR detection. The possible target pixels in the background window which have an effect on background statistics can be removed by sliding windows detection. Then, the $\mathrm{K}$ distribution probability density function (referred to as $f_{2}(x)$ in the paper) is obtained by making a statistic for the rest pixels in background window with $\mathrm{K}$ distribution model. Finally, the local threshold $\mathrm{T}_{\mathrm{G}}$ can be calculated out by setting the second level false alarm rate (referred to as $\mathrm{P}_{\mathrm{fa} 2}$ in the paper) and solving the equation 3.5.

\subsection{The Fast Algorithm}

Though the detection speed of two-parameter CFAR algorithm is improved by compared with the adaptive algorithm based on K distribution, it still runs slowly when it comes to the detection of high-resolution and large-size SAR images, due to the large data calculation when solving the parameters of $\mathrm{K}$ distribution model. The main reason for the low speed of two-parameter CFAR algorithm for local detection is that there are too many targets detected in global detection. In order to improve the detection speed of the two-parameter CFAR algorithm, the local detection speed needs to be improved. In this paper, the two methods that called prescreening ship pixels and multithreading technology are adopted to improve the speed of local detection.

1) Prescreening ship pixels

Generally speaking, the distance between two ships is at least twice as long as the ship, so there will not be any other ships in a ship within the scope of a ship's length. The marked pixels are detected in second level CFAR detection from left to right, and from top to bottom. When a marked pixel is identified as the ship pixel by local detection, we can think the marked points whose pixel value are greater than its value in the range of one ship's length around it can be directly screened out as ship pixels, which don't need to be detected in sliding window. In this way, we can decrease the use of $\mathrm{K}$ distribution and improve the detection speed.

2) Multithreading technology

Multithreading is a process that allows an application to have two or more threads at the same time to support transactional concurrency and multitasking. Multithreading technology is a technology to realize the concurrent execution of multiple threads from the software or hardware. The speed of local detection can be greatly improved if we can divide the SAR image into several parts and adopt multithreading technology to detect these parts at the same time, so multithreading technology is adopted to improve the detection speed in this paper.

Many experiments show that it is not only easier to calculate, but also more reasonable when the large-size SAR image after global detection is divided into several rectangle regions. The improved speed is not obvious if one SAR image is divided into two rectangle regions. The targets distribution is easy to be inhomogeneous if one SAR image is divided into three rectangle regions. It is too complicated and difficult if one SAR image be divided into more than four rectangle regions. Therefore, as is shown in Figure 3, the SAR image is equally divided into four rectangle regions, then, make local detections for the target pixels in four regions at the same time by multithreading technology, making the distribution relatively homogeneous and the speed higher, which is more reasonable. 




Figure 3. Quartering the SAR Image

However, if the marked pixels distribution after global detection is extremely inhomogeneous, which makes a big difference among the number of marked pixels in the four rectangle regions divided equally, at this time, the speed can't be improved much even if adopting multithreading technology. Therefore, the detection speed should be improved by redistributing the SAR image.

Detailed ways are as follows: first, make statistics for the number of all marked pixels in SAR image and the average number of four regions, separately referred to as $\mathrm{N}_{\mathrm{t}}, \mathrm{N}_{\mathrm{m}}$ $\left(\mathrm{N}_{\mathrm{t}}=8361, \mathrm{~N}_{\mathrm{m}}=2091\right)$ after the global computing. Second, the SAR image is divided into four rectangle regions, separately referred to as A1, A2, A3, A4, which is shown in in Figure 4(a), accordingly, the number of marked pixels in four regions referred to as $\mathrm{N}_{1}, \mathrm{~N}_{2}, \mathrm{~N}_{3}, \mathrm{~N}_{4}$ separately $\left(\mathrm{N}_{1}=1533, \mathrm{~N}_{2}=1907, \mathrm{~N}_{3}=709, \mathrm{~N}_{4}=4382\right)$. If $\mathrm{N} 4$ is the largest among those, the region owing the most marked pixels is A4.Comparing N4 with $2 \times \mathrm{N}_{\mathrm{m}}$, If $\mathrm{N} 4$ is greater than $2 \times \mathrm{N}_{\mathrm{m}}$, redistributing the four regions. Comparing the number of marked pixels in A2 region with that in $A 3$ region neighboring $A 4$ region, if $N_{2}>N_{3}$, A4 and $A 3$ regions should be redistributed, and remove the area of 1/3 from A3 region to A4 region, if not, A4 and A2 regions should be redistributed. The redistributed result is shown in Figure 4 (b).

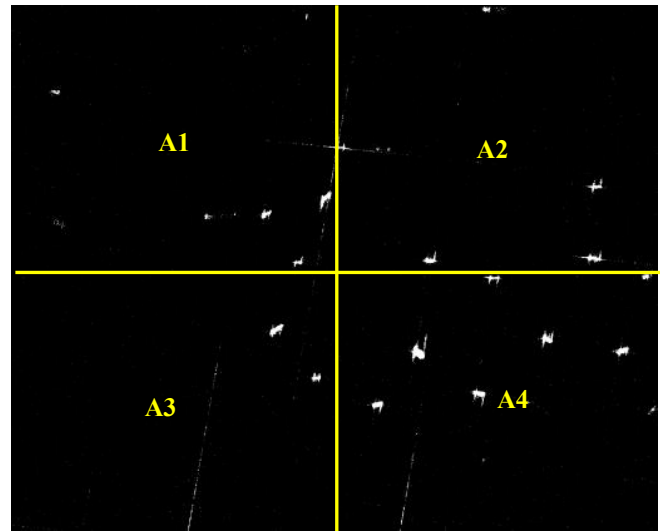

(a) The quartered SAR image

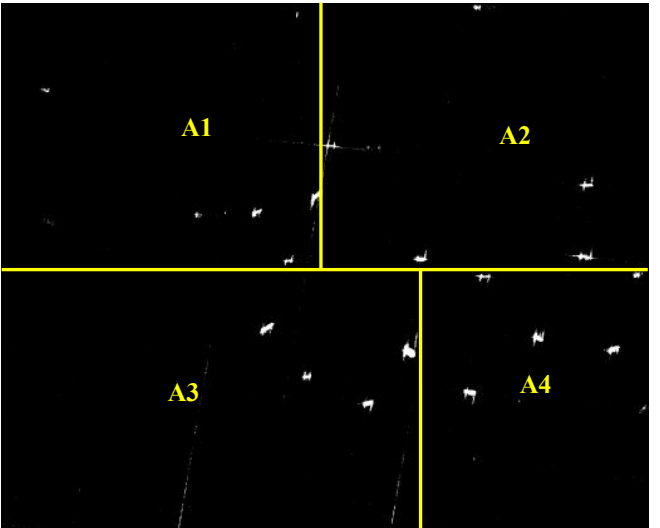

(b) The redistributed SAR image

Figure 4. Redistributing the SAR Image

After four regions are redistributed, the marked pixels distribution is relatively homogeneous, then, multithreading technology is adopted to detect the redistributed four regions at the same time, so as to improve the speed of local detection. Finally, we will splice the four regional detection results together into a complete SAR image. 


\section{The Analysis of the Detection Results}

Two SAR images are adopted to analyze the detection results. Figure 5(a) shows an airborne VV polarization single-look SAR image, with a resolution of $3 \times 3$ meters and a size of $1517 \times 1864$ pixels, taken by a high resolution radar satellite COSMO_SkyMed in 2007, with the GCS_WGS_1984 coordinate system, geographical coordinates for longitude $121.96^{\circ}$ latitude $38.96^{\circ}$, which has 20 ship targets. Figure $6(\mathrm{a})$ shows an airborne $\mathrm{HH}$ polarization single-look SAR image, with a resolution of $3 \times 3$ meters and a size of $1541 \times 1866$ pixels, taken by a high resolution radar satellite TSX1_SAR in 2007, with the GCS_WGS_1984 coordinate system, geographical coordinates for longitude $119.55^{\circ}$ latitude $35.06^{\circ}$, which has 9 ship targets. Two SAR images are detected by the proposed fast algorithm and the two-parameter CFAR algorithm, then, the detection results are analyzed by studying comparatively detection accuracy and speed of the two algorithms. The sliding window size in the experiment is determined by the target pixel size and image resolution. For Figure 5(a) and Figure 6(a), the target window size $1 \times 1$, the protected window size $301 \times 301$, the background window size is $333 \times 333$, and the first false alarm rate $\mathrm{P}_{\mathrm{fa}}$ is set to $4 \mathrm{e}-3$ when solving global threshold, the second false alarm rate $\mathrm{P}_{\text {fa2 }}$ set to $1 \mathrm{e}-3$ when solving local threshold.

After the global threshold is calculated out by histogram distribution, the target pixels can be screened out rapidly in SAR image. Because the histogram statistic records the real distribution of all the pixels in SAR image, and the false alarm rate is set high, it can ensure that the screening results contain all target pixels. As are shown in Figure 5(b) and Figure 6(b), 10823 and 11602 targets are screened out separately. Then, both Figure 5(b) and Figure 6(b) are detected simultaneously in sliding window by multithreading technology after divided into four rectangle regions. Finally, the ships are screened out by making the four-connected neighborhood area statistics to obtain the detection result images.

The detection results of the two algorithms are shown in Figure 5(c), Figure 5(d) and Figure 6(c), Figure 6(d). The detection time is separately 540 seconds, 69 seconds and 596 seconds, 86 seconds on the same computer, and the number of detected ship is separately 18, 20 and 9, 11. By the comparison of Table 1 and Table 2, the detection speed and accuracy of the fast algorithm proposed in this paper both are greatly better than that of the two-parameter CFAR algorithm. Therefore, the fast algorithm proposed in this paper is more suitable to apply to the ship detection system.

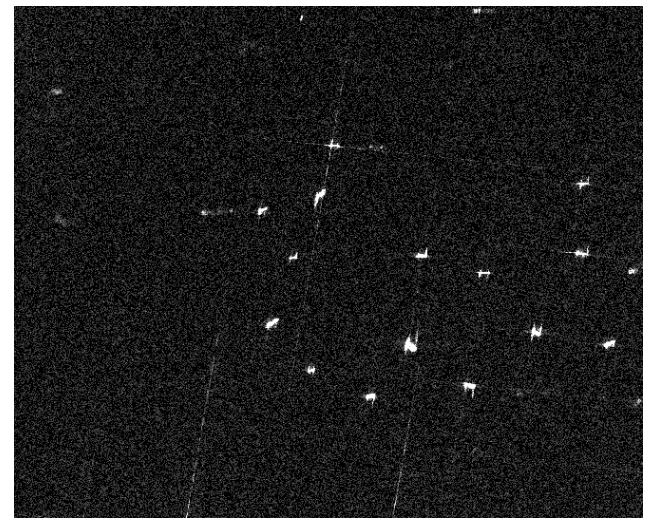

(a) Original SAR image

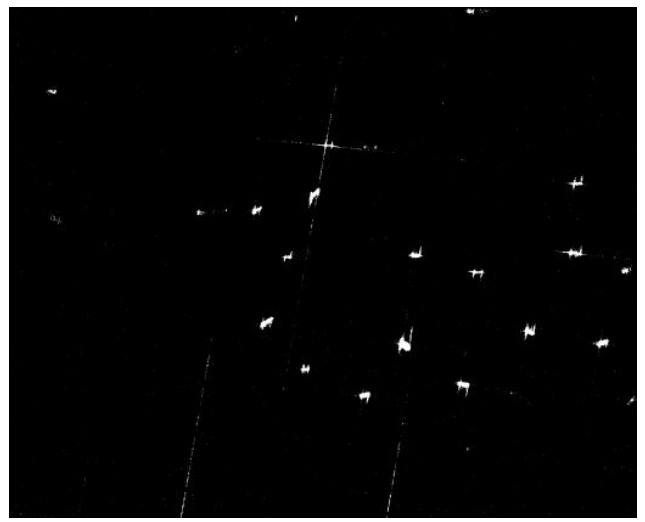

(b)Global detection result 

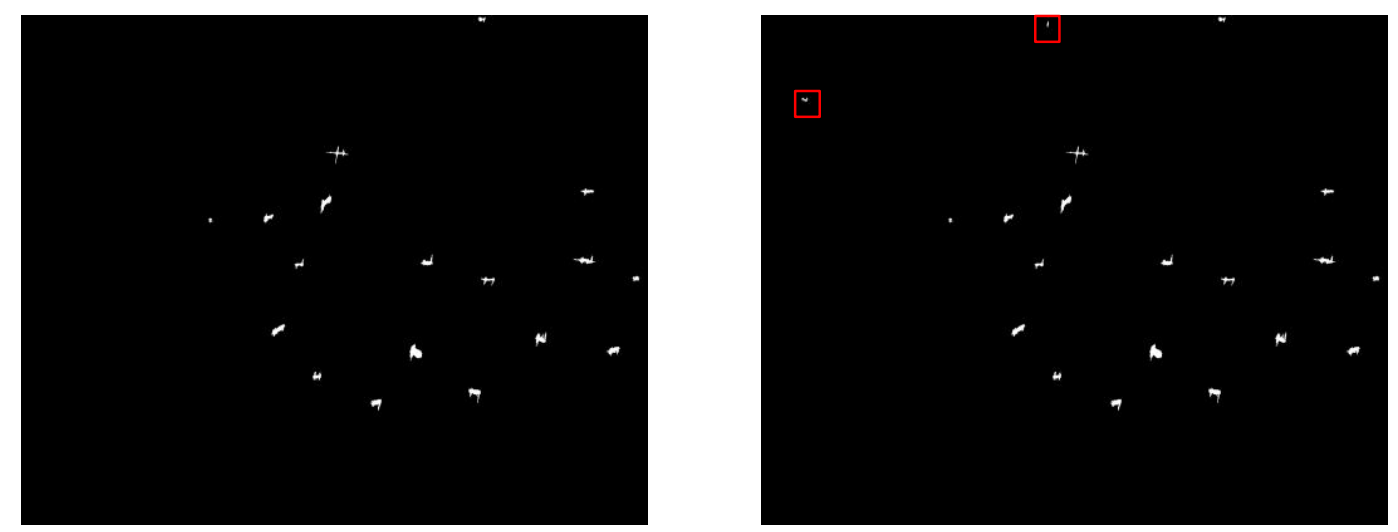

(c) Two-parameter CFAR algorithm detection result

(d) Fast algorithm detection result

Figure 5. Detection Results of the Two Algorithms for COSMO-SkyMed Satellite Image

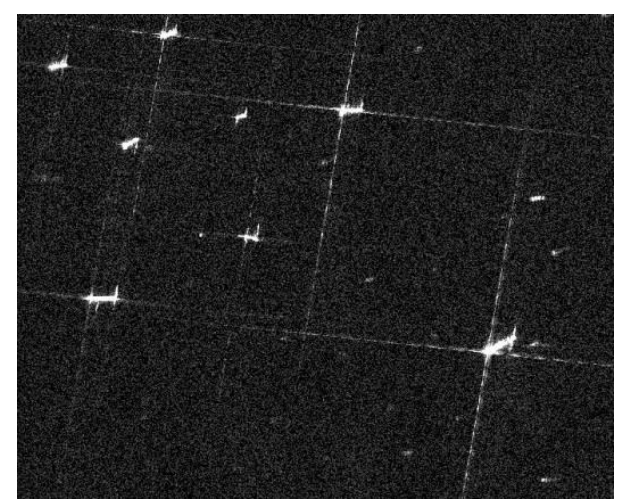

(a) Original SAR image



(c) Two-parameter CFAR algorithm detection result (d) Fast algorithm detection result

Figure 6. Detection Results of the Two algorithms for TSX1_SAR Satellite Image 
Table 1. Performance comparisons between the two algorithms for COSMO-SkyMed satellite image

\begin{tabular}{|c|c|c|c|c|c|c|}
\hline Two algorithms & $\begin{array}{c}\text { Number } \\
\text { of all } \\
\text { ships }\end{array}$ & $\begin{array}{c}\text { Number of } \\
\text { marked pixels } \\
\text { in local } \\
\text { detection }\end{array}$ & $\begin{array}{c}\text { Number of } \\
\text { detected } \\
\text { ships }\end{array}$ & $\begin{array}{c}\text { False } \\
\text { alarm }\end{array}$ & $\begin{array}{c}\text { Missed } \\
\text { alarm }\end{array}$ & $\begin{array}{c}\text { Running } \\
\text { time }\end{array}$ \\
\hline $\begin{array}{c}\text { The two-parameter } \\
\text { CFAR algorithm }\end{array}$ & 20 & 10823 & 18 & 0 & 2 & $540 \mathrm{~s}$ \\
\hline The fast algorithm & 20 & 10823 & 20 & 0 & 0 & $69 \mathrm{~s}$ \\
\hline
\end{tabular}

Table 2. Performance comparisons between the two algorithms for TSX1_SAR satellite image

\begin{tabular}{|c|c|c|c|c|c|c|}
\hline Two algorithms & $\begin{array}{c}\text { Number } \\
\text { of all } \\
\text { ships }\end{array}$ & $\begin{array}{c}\text { Number of } \\
\text { marked pixels } \\
\text { in local } \\
\text { detection }\end{array}$ & $\begin{array}{c}\text { Number of } \\
\text { detected } \\
\text { ships }\end{array}$ & $\begin{array}{c}\text { False } \\
\text { alarm }\end{array}$ & $\begin{array}{c}\text { Missed } \\
\text { alarm }\end{array}$ & $\begin{array}{c}\text { Running } \\
\text { time }\end{array}$ \\
\hline $\begin{array}{c}\text { The two-parameter } \\
\text { CFAR algorithm }\end{array}$ & 9 & 11602 & 11 & 2 & 0 & $596 \mathrm{~s}$ \\
\hline The fast algorithm & 9 & 11602 & 9 & 0 & 0 & $86 \mathrm{~s}$ \\
\hline
\end{tabular}

\section{Conclusion}

In this paper, an adaptive and fast CFAR algorithm based on multithreading for ship detection in SAR images is proposed, which is divided into global and local detection. The global threshold filtering detection greatly reduces the number of ships target pixels. The rest ones need to be detected accurately in local detection. The multithread-based parallel computing is adopted in the local detection, which improves the speed of the detection algorithm greatly once again. The final detection results show that the fast algorithm in this paper is more suitable to apply to the ship detection system, because its detection accuracy and speed both are greatly improved by compared with the two-parameter CFAR algorithm.

\section{Acknowledgement}

This paper is supported by fund project "Global change and air-sea interaction" of China, and the project number is GASI-02-YG-BC01. We thank the fund project and colleagues in our laboratory sincerely for their valuable comments and help.

\section{References}

[1] Biao Hou, Xingzhong Chen, and Licheng Jiao, "Multilayer CFAR Detection of Ship Targets in Very High Resolution SAR Images", IEEE Geoscience and Remote Sensing Letters, vol. 12, no. 4, (2015), pp.811-815.

[2] R. Riflcin, "Analysis of CFAR performance in Weibull clutter", IEEE Transactions on Aerospace and Electronic Systems, vol. 30, no. 2, (1994), pp.315-329.

[3] A.C. Frery, H. J. Muller, C. C. F. Yanasse, and S.J.S. Sant'Anna, "A model for extremely heterogeneous clutter", IEEE Transactions on Geoscience and Remote Sensing, vol. 35, no. 3, (1997), pp. 648-659.

[4] S.Erfanian, and V. T. Vakili, "Introducing excision switching CFAR in K distributed sea clutter", Signal Processing, vol. 89, no. 6, (2009), pp. 1023-1031.

[5] S. Kuttikkad and R. Chellappa, "Non-Gaussian CFAR techniques for target detection in high resolution SAR images", in Proc. ICIP, vol. 1, (1994), pp. 910-914. 
[6] Jiayuan Liu, and Shuhong Jiao, "The study of algorithm for ship detection in SAR image", Mater Dissertation Research Paper, Harbin institute of technology. China, (2013).

[7] M. E. Smith and P K. Varshney, "Intelligent CFAR processor based on data variability", IEEE Trans. Aerosp. Electron. Syst., vol. 36, no. 3, (2000), pp. 837-847.

[8] J. S. Salazar, II, "Detection schemes for synthetic aperture radar imagery based on a beta prime statistical model", Ph.D. dissertation, Univ. New Mexico, Albuquerque, (1999).

[9] M. di Bisceglie, and C. Galdi, "CFAR detection of extended objects in high-resolution SAR images", IEEE Transactions on Geoscience and Remote Sensing, vol. 43, no. 4, (2005), pp. 833-843.

[10] G. Gao, G. Kuang, Q. Zhang, and D. Li, "Fast detecting and locating groups of targets in high-resolution SAR images," Pattern Recognit., vol. 40, no. 4, (2007), pp. 1378-1384.

[11] G. Gao, L. Liu, L. Zhao, G. Shi, and G. Kuang, "An Adaptive and Fast CFAR Algorithm Based on Automatic Censoring for Target Detection in High-Resolution SAR Images", IEEE Transactions on Geoscience and Remote Sensing, vol. 47, no. 6, (2009), pp.1685-1696.

[12] Wentao An, Chunhua Xie, and Xinzhe Yuan, "An Improved Iterative Censoring Scheme for CFAR Ship Detection With SAR Imagery", IEEE Transactions on Geoscience and Remote Sensing, vol. 52, no. 8, (2014), pp.4585-4895.
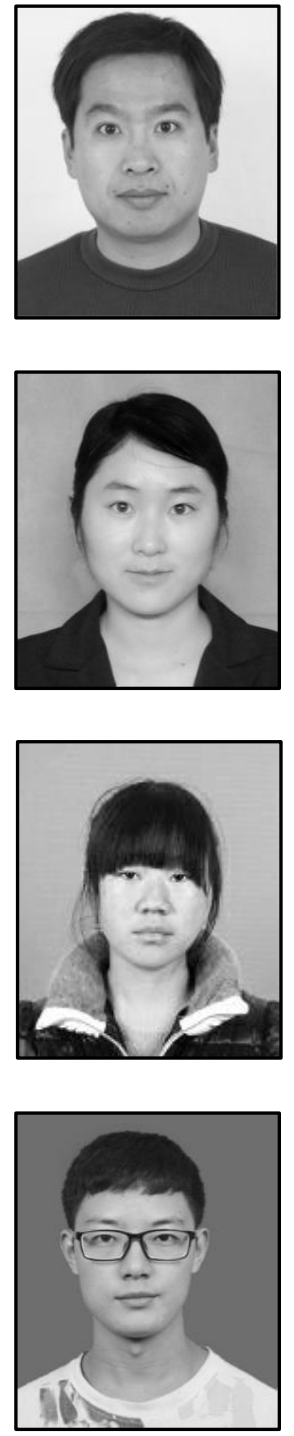

\section{Authors}

Ruifu Wang, received the B.S degree in Cartography and Geography Information System from NanJing University in 1999, the MS degree in Physical Oceanography from the First Institute of Oceanography, SOA, PRC in 2003, the $\mathrm{PhD}$ degree in Environmental Science in Ocean University of China in 2006. His current research interest is in the area of GIS marine application and integrated Electronic Chart on GIS.

Jie Li, received B.S in Surveying and Mapping Engineering from Shandong University of Science and Technology in 2014. Now she is a graduate student in Shandong University of Science and Technology. Her current research interests include management, visualization and analysis of spatial data, and secondary development based on GIS.

Yingjie Hao, received B.S in Surveying and Mapping Engineering from Shandong Datong University in 2014. Now she is a graduate student in Shandong University of Science and Technology. Her current research interests include management, visualization and analysis of spatial data, and secondary development based on GIS.

Jiagui Li, received B.Sc in Geographic Information System from Shandong University of Science and Technology in 2015. Now she is a graduate student in Shandong University of Science and Technology. Her current research interests include management, visualization and analysis of spatial data, and secondary development based on GIS. 
International Journal of Hybrid Information Technology

Vol. 10, No. 8 (2017) 